\title{
THE TRANSPLANTATION OF NUCLEI BETWEEN TWO SUBSPECIES OF XENOPUS LAEVIS
}

\author{
J. B. GURDON \\ (Beit Memorial Research Fellow) \\ Department of Zoology, Parks Rood, Oxford
}

\section{INTRODUCTION}

Received 25.iii.6r

THE technique of nuclear transplantation enables an embryonic nucleus from one kind of frog to be combined with the egg-cytoplasm from another kind. The extent to which the resulting transplantembryos can differentiate varies according to how closely related are the species or subspecies from which the nucleus and cytoplasm are derived. Embryos, tadpoles, and frogs derived from any combination of nucleus and cytoplasm from different species or subspecies are described as "heterotypic". In the experiments described below, nuclei have been transplanted between two subspecies of Xenopus laevis, which are sufficiently closely related for some of the resulting transplant-embryos to grow into adult frogs. These show the result of interaction between the nucleus and cytoplasm during the differentiation of the characters which distinguish the subspecies. The transfer of nuclei from advanced donor stages into egg-cytoplasm of the foreign subspecies indicates how similar is the rate, and time of onset, of nuclear differentiation in the two subspecies; these results also indicate the significance of the considerable difference in these respects between Xenopus laevis and Rana pipiens (Gurdon, I960c). Lastly, frogs derived from the serial transfer of nuclei between the subspecies show whether nuclei undergo autonomous differentiation or suffer any damage as a result of continued transplantation.

\section{MATERIAL AND METHODS}

The frogs used in these experiments belong to the two subspecies Xenopus laevis laevis (Daudin) and Xenopus laevis victorianus Ahl. The stock individuals of $X . l$. victorianus were reared in the laboratory from tadpoles kindly made available by Professor Newth, and obtained from Entebbe, Uganda. The X.l. laevis frogs used were reared in the laboratory from specimens originally obtained in S. Africa. The main differences between the subspecies concern size and colour pattern, and are described below (p. 308). Hybrids obtained by reciprocal crosses between the two subspecies are viable and fertile, and more or less intermediate in character (Blackler, Fischberg and Newth, in preparation).

Throughout these transplantation experiments, donor nuclei from the vegetal hemisphere or endoderm region have been used since these are easier to handle than nuclei from other embryonic regions. Eggs were obtained by injecting females with gonadotropic hormone.

The transplantation technique employed has been described elsewhere (Elsdale, 
Gurdon and Fischberg, 196o ; Gurdon, 196o $b$ ). The technique involves the injection of an embryonic cell, the wall of which has been broken, into an unfertilised and enucleated egg. It mainly differs from that of Briggs and King (1953) in two respects. First the recipient eggs of Xenopus are irradiated with ultraviolet for about one minute. This treatment kills the egg-nucleus which then degenerates in the egg-cytoplasm, but the latter is not damaged in any way (Gurdon, I96oa). Briggs and King, on the other hand, remove the egg-nucleus with a needle from the recipient eggs of Rana pipiens. Secondly, the Xenopus nuclear marker (Elsdale, Fischberg and Smith, I958) was used, which provides proof that only the injected nucleus has participated in the development of the transplant-embryo. The marker behaves as a mutation which suppresses the formation of the nucleolus from one haploid set of chromosomes ; the application of its use for transplantation experiments has been described elsewhere (Elsdale, Gurdon and Fischberg, 1960).

\section{RESULTS}

(i) The early development of heterotypic transplant-embryos

Text-figs. I and 2 consist of camera lucida drawings of donor embryos and the transplant-embryos derived from them. In these experiments nuclei from both subspecies have been transplanted into recipient eggs of laevis; all transplantations in text-fig. I were made into the eggs of one frog, and all those in text-fig. 2 into the eggs of another. Twentythree nuclei were transplanted from each donor embryo, but only those transplant-embryos are shown which became regular late blastulae. Those transplantations which failed to cleave at all or which cleaved irregularly are not shown, since this abnormality may be due, in some cases at least, to the technical treatment connected with transplantation (Gurdon, I $960 b$ ). The number of embryos underneath each donor therefore represents the proportion of transplanted nuclei out of 23 which gave rise to regular late blastulae. Transplant-embryos were not kept beyond the early feeding tadpole stage, since it has been found from previous experience that at least 95 per cent. of embryos which reach this stage can be easily reared into adult fertile frogs. In these diagrams, therefore, at least 95 per cent. of all feeding tadpoles represent the presence in the original donor embryo of undifferentiated and totipotent nuclei.

Text-fig. I shows the results of transplanting blastula and gastrula nuclei. The embryos derived from laevis mid-gastrula nuclei are all normal, and those in the other two series are nearly all normal. This is the kind of result that has previously been obtained from undifferentiated nuclei of laevis blastulae and gastrulae (Gurdon, I960c). The conclusion from these experiments is that there is no difference in the developmental capacity of early donor stage nuclei of laevis and victorianus, and hence that blastula and gastrula nuclei of both subspecies are still undifferentiated.

Text-fig. 2 illustrates experiments involving the transplantation of nuclei from more advanced donor stages of laevis and victorianus. The quality of eggs laid by frogs kept in the laboratory is very variable, and it is necessary to find out whether abnormal transplant-embryos represent differentiated nuclei or merely recipient eggs incapable of 
normal development. In text-fig. 2, the embryos derived from stage 28 nuclei of laevis are nearly all normal. As the eggs laid by a frog at any one ovulation are usually of consistent quality, this indicates that the abnormalities obtained after transfer of nuclei from the stage 37 and older donor stages are in this case due to nuclear differentiation and not to the poor quality of the recipient eggs. Nuclei from hatched tadpoles of both subspecies (Ist, 2nd and $5^{\text {th }}$ series of text-fig. 2) gave very similar results.

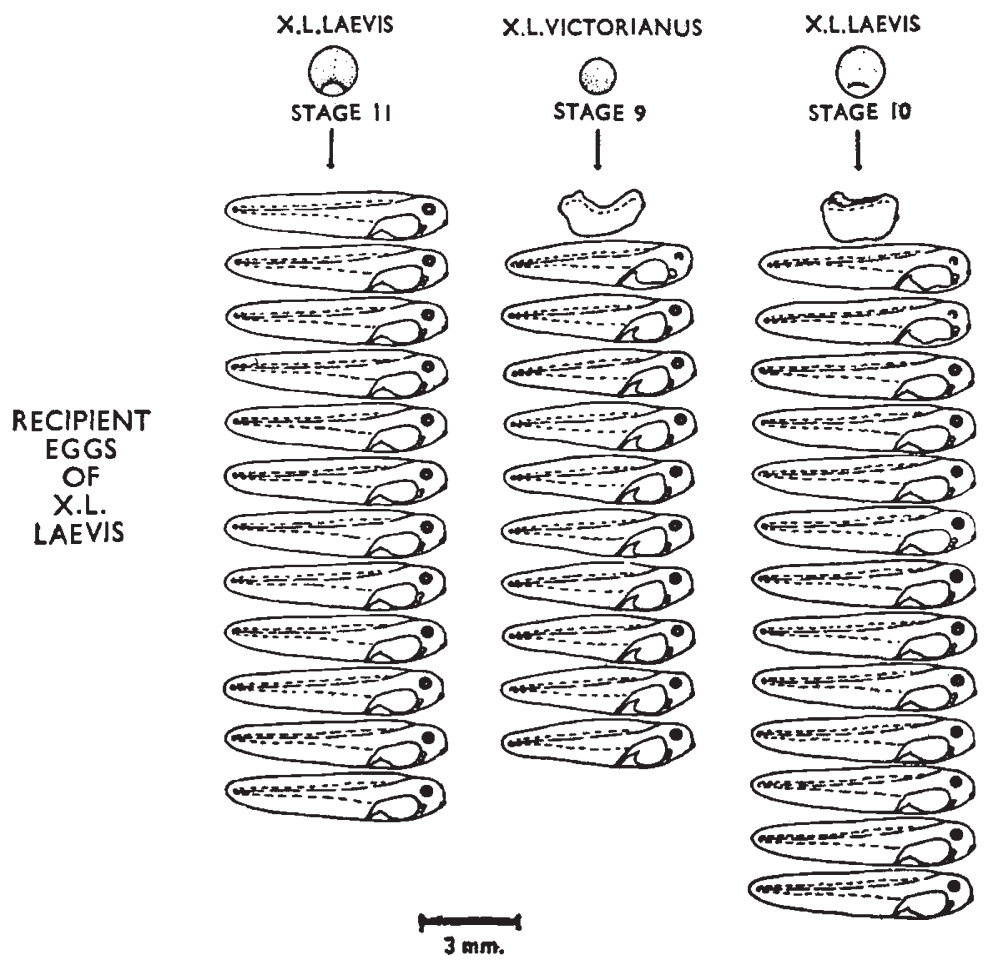

TeXT-pIG. I.-Camera lucida drawings of embryos derived from the transplantation of nuclei from a mid-gastrula (laevis), a late blastula (victorianus), and an early gastrula (laevis), all into recipient eggs laid by one laevis female. Twenty-three transplantations were made in each of the three series, but only those transplant-embryos which cleaved regularly are shown.

Two conclusions can be drawn from the experiments illustrated in text-figs. I and 2. First, the rate of differentiation of endoderm nuclei is very similar in both subspecies. Second, there is no incompatibility preventing the nuclei of one subspecies from cleaving regularly and forming normal tadpoles when combined with the cytoplasm of the other subspecies.

Reciprocal experiments have also been carried out in which nuclei from early and advanced stages of both subspecies were transplanted into recipient eggs of victorianus. It was found that the nuclei of both subspecies gave very similar results, but that the development of transplant-embryos from all donor stages was much more abnormal 
with the recipient eggs of victorianus than with those of laevis. This result could be due to the variable quality of victorianus eggs, and perhaps also to their smaller size (I mm. diameter, compared to I. $5 \mathrm{~mm}$. for laevis eggs). On the other hand it might indicate that the development of transplant-embryos varies according to the type of recipient egg used. It is not yet possible to distinguish between these possibilities, since the smaller number of eggs laid, over a shorter period of time, by victorianus females made it impossible to obtain adequate control series, with which to assess egg-quality.
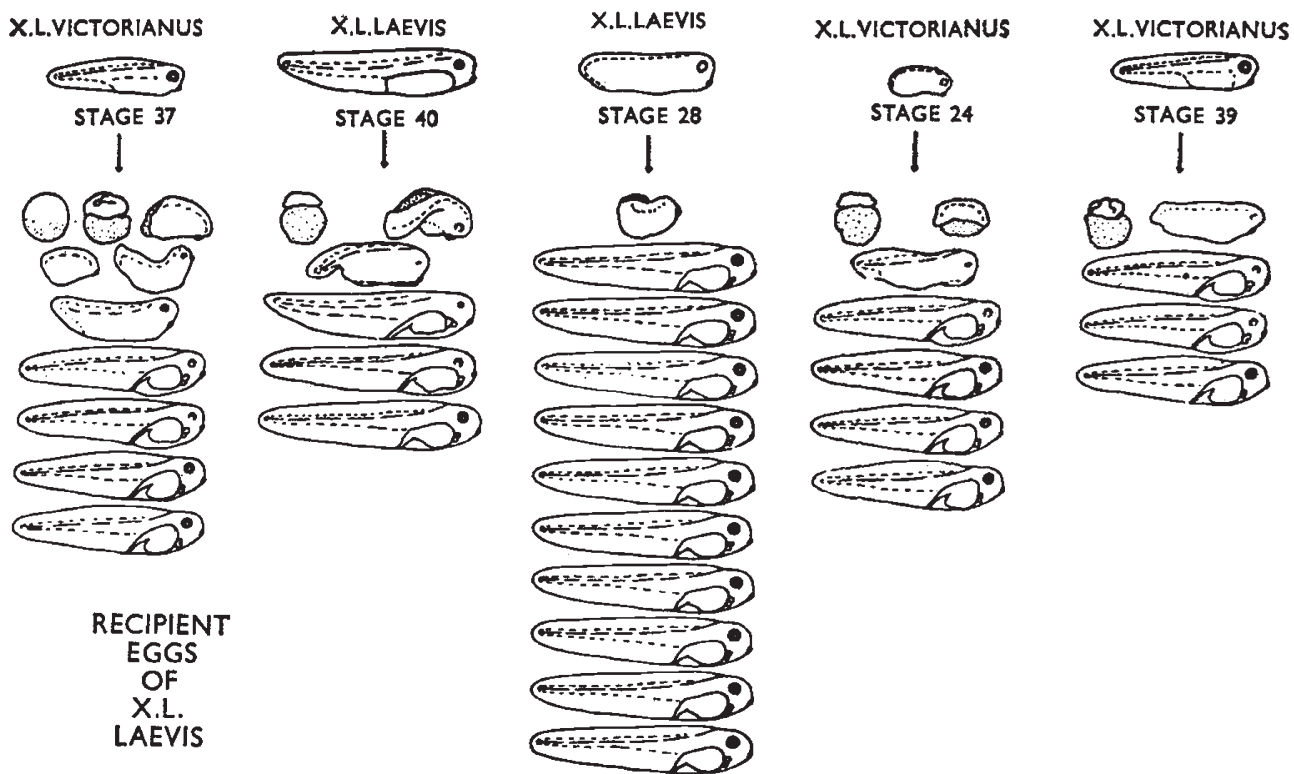

$3 \mathrm{~mm}$.

TExT-FIG. 2.- See legend to text-fig. 1 , which is directly applicable, except that donor nuclei were taken from the endoderm of a hatched tadpole of victorianus, a swimming tadpole and a tail-bud stage of laevis, and a post-neurula and swimming tadpole of victorianus. Again 23 transplantations in each series.

(ii) The differentiation of subspecific characters in heterotypic transplant-animals

Plate IA shows certain stages in the development of victorianus controls (left-hand column), laevis controls (right-hand column), and the two heterotypic combinations (centre columns). The top row (A) consists of blastulae which indicate the original egg size. Victorianus eggs are less than $\frac{1}{3}$ of the volume of, and much less pigmented than, laevis eggs, and this difference in size persists up to the time when the tadpoles begin feeding (row F). This shows that a nucleus can participate in normal development with an initially much greater or much smaller volume of cytoplasm than is usual for it. 
Apart from this difference in size, the first clear distinction between the subspecies concerns the appearance of body melanophores; in laevis these appear before hatching (row G), while in victorianus they do not appear until the swimming tadpole stage (row E). In the heterotypic combinations the appearance of these melanophores is entirely nucleus-controlled. They can be seen before hatching in laevis nuclei with victorianus eggs, but do not appear until the swimming tadpole stage in victorianus nuclei with laevis eggs (row E). Another clear difference between the subspecies is the stage at which anal melanophores appear. These melanophores are first seen in victorianus very soon after feeding commences (Nieuwkoop and Faber's (1956) stage 48-49), while in laevis they do not appear until the tadpoles have already been feeding for I to 2 weeks (stage 57). In heterotypic tadpoles this character again develops just as in the subspecies which contributed the nucleus. Other differences between the subspecies are described elsewhere (Blackler, Fischberg and Newth, in preparation). In tadpoles, these concern the posterior edge of the endoderm at stage 40, the angle between the anal tube and gut at stage 4I, and the shape and depth of the tail fin in advanced feeding tadpoles. At metamorphosis there is a clear difference in the shape of the resorbing tail, and later in the shape of the forearms. After metamorphosis the colour and pattern of the two types of frogs is quite distinct. Victorianus frogs are a light yellowish-green, and gradually acquire a very fine freckling of dark green or black as they reach maturity. Laevis frogs, on the other hand, have a darker green or blackish ground colour, with pronounced black mottling. As victorianus reach maturity, the underside of the hind-legs develops a yellow coloration which is bordered on the outer side by an irregular band of blackish spots. This pattern is not present in laevis. The latter are much bigger frogs than victorianus; when adult, the average weight of laboratory reared laevis females and males is 75 and 45 grams respectively, while that of victorianus females and males is 15 and $8 \frac{1}{2} \mathrm{gms}$. In all these respects the heterotypic embryos, tadpoles, and frogs are indistinguishable from controls of the subspecies from which their nuclei were derived.

Plate II contains photographs of adult frogs, all of which are males. Frogs derived from victorianus nuclei and laevis cytoplasm (top left) are identical to control victorianus (lower left), and show none of the characters of the laevis control (middle left). The right-hand side of the plate shows a serial transplantation experiment. Original donor nuclei from a victorianus late gastrula were transplanted into laevis recipient eggs. When the resulting embryos became late gastrulae, one of them was used to provide nuclei for further transplantations into other laevis eggs. The remaining gastrula transplant-embryos were allowed to develop further, some of them becoming adult frogs (as shown). This procedure was repeated three times so that the nuclei that have given rise to the three frogs shown at the bottom 
right-hand side of the picture have had to synthesise and replicate in foreign cytoplasm for four "gastrula generations "- that is, for over 6 o divisions or for 45 divisions in excess of those that take place in the normal development of an individual. In spite of this, the nuclei have been entirely unaffected by the foreign cytoplasm, since the $4^{\text {th }}$ transfer frogs are indistinguishable from victorianus controls. These $4^{\text {th }}$ transfer frogs (bottom right) were mated to control victorianus females; they proved to be as fertile as controls reared in the laboratory and their offspring were entirely of the victorianus type. This constitutes further evidence that the nuclei were unaffected by the foreign cytoplasm, since the male gametes of these frogs carried none of the laevis characters; the latter should be easily observable since hybrids between laevis and victorianus are intermediate in character, as mentioned above.

It should be mentioned here that the adult frogs shown in plate II have been selected as the largest individuals obtained. In the transplantation series concerned, other frogs were also obtained, but these only differed from those shown in that they were smaller. The retardation of their growth seems clearly to be a consequence of the laboratory rearing conditions (Gurdon, I96I). The selection of frogs for plate II is therefore only a question of having chosen those individuals which matured first, and does not in any way affect the conclusions.

A further series of experiments have been carried out in which nuclei from original laevis donors were transferred to victorianus cytoplasm; some of these were then serially transferred back to laevis eggs for 2 gastrula generations, while others were transferred again into victorianus eggs. At each stage in the experiment, some transplantembryos were kept for rearing up into adult frogs, the most fully grown of which are shown in plate Iв. Irrespective of the number of divisions that their nuclei have made in foreign cytoplasm, or in their own kind after back-transfer, the resulting frogs were typical laevis in every respect. This confirms the results above, that there is, with very rare exceptions, complete nuclear specificity in the expression of all distinguishing characters so far studied in these two subspecies.

\section{DISCUSSION AND CONCLUSIONS}

(i) The differentiation of distinguishing characters in individuals resulting from heterotypic combinations of nucleus and cytoplasm

No attempt will be made to review the literature concerning this question but one or two examples will be quoted to indicate the main kinds of results that have been obtained. This problem has been extensively investigated by fertilising enucleated eggs with sperm from a different species or subspecies; the resulting embryos, which are haploid, die at an early embryonic stage characteristic for the particular combination concerned (Baltzer, 1952). It is only with relatively closely related forms that such hybrid merogons are at all viable, but the distinguishing characters do not usually become evident 
before the tadpoles die owing to their haploid condition. This difficulty has been partially overcome by transplanting certain tissues from the haploid hybrid merogon to a normal diploid host, where they can differentiate further, showing up specific characters (e.g. Hadorn, 1937; Dalton, 1946). Most of the characters which have been studied by these means differentiate just like those of the species or subspecies which provided the nucleus. On the other hand cytoplasmic control of the expression of certain characters has also been recorded (Hadorn, 1937).

In more recent years, this general problem has been investigated using the technique of nuclear transplantation which avoids the inviability associated with the haploid condition. In the case of nuclear transfers between different species or genera, the resulting transplant-embryos have always been unable to survive up to a stage when distinguishing characters become apparent. However, Sambuichi (1957) transplanted nuclei between two subspecies of Rana nigromaculata and found that in all characters observed the heterotypic transplant-embryos developed like the "nuclear subspecies"; McKinnell (I960) also observed an entirely nucleus-specific expression of a single character by which two races of Rana pipiens differ.

The experiments with Xenopus subspecies described above are consistent with previously published results, in that all the characters studied which affect embryonic and larval development as well as adult frogs were controlled entirely by the nucleus and not by the cytoplasm. Only direct consequences of the volume of egg-cytoplasm resulted in a cytoplasmic effect on development. It is not certain whether the egg-cytoplasm of the two subspecies is qualitatively different or only quantitatively so. However, the fact that the nucleus of one subspecies is able to express itself perfectly in foreign cytoplasm shows that there is no nucleo-cytoplasmic incompatibility between the subspecies, and that the nucleus is not affected in its function by such differences as do exist in the two kinds of egg-cytoplasm.

Another matter, to which these results are relevant, concerns what effect, if any, foreign cytoplasm has on nuclei synthesising from it. Astaurov and Ostriakova-Varshaver (1957) have made diploid hybrid merogons between two types of silk-moth and have then back-crossed them to the same type as that which provided the nucleus of the merogon. In this way they exposed the nuclei of one type to the cytoplasm of the other for one whole generation, but found that they had been entirely unaffected by this.

By means of serial nuclear transplantation in Anura it is possible to transfer a nucleus to foreign cytoplasm and then, when it has synthesised there for several mitoses, to return it to its own kind of cytoplasm. A comparison of the resulting back-transfer embryos with controls whose nuclei have been transferred twice (for two gastrula generations) into eggs of the same kind, shows whether nuclei have been affected by their sojourn in foreign cytoplasm. When 
nuclei are transferred to eggs of another species, the heterotypic combination (Ist out-transfer) usually dies at the late blastula or early gastrula stage owing to the nucleo-cytoplasmic incompatibility. When these nuclei are back-transferred (before developmental arrest) to their own kind of cytoplasm, they again cease development at an early stage, showing that they have been affected by the foreign cytoplasm (Fischberg, Gurdon and Elsdale, I958; Moore, 1958). The present experiments show that nuclei transferred to the eggcytoplasm of a foreign subspecies give rise to normal development; they also show that such nuclei are entirely unchanged when transferred back to their own kind of eggs. This still holds true even when they have been exposed to foreign cytoplasm for over 60 mitoses, and also when returned to their own kind of eggs after at least 30 mitoses in foreign cytoplasm. It must be mentioned here that occasional individuals are intermediate between the nuclear and cytoplasmic subspecies for one character or another. However, these cases are very exceptional and are being investigated further.

\section{(ii) The effect on transplant-embryo development of the dying irradiated nucleus of Xenopus eggs}

Another consequence of these experiments concerns the problem of the difference between Xenopus and Rana in the potentialities of endoderm nuclei. The rate and time of onset of nuclear differentiation is about the same in $X . l$. victorianus and $X$.l. laevis-that is, there is still a small proportion of undifferentiated nuclei in the hatched tadpole gut of Xenopus. This is in strong contrast to the earlier onset and much faster rate of nuclear differentiation in Rana pipiens, in which almost no endoderm nuclei are able to develop normally after transplantation from the tail-bud stage (Briggs and King, 1957). It has been pointed out (Gurdon, Elsdale and Fischberg, I958; Briggs and King, 1960) that this difference could be partially due to a reversal or repair of differentiation in nuclei from advanced donor stages of Xenopus. It is possible that hatched tadpole nuclei are all differentiated, but that some become totipotent again, after transplantation to uncleaved eggs. Totipotency might be regained if the transplanted nucleus were to absorb from the dying egg-nucleus certain substances for which it had become deficient in the course of differentiation. It is not necessary that this reversal should be an exact retracing of the steps of differentiation; it might consist only of a compensation for the differentiated state of the nucleus by the inclusion in it of other chromosome fragments. Such a process might take place in Xenopus and not in Rana, since in Xenopus the nucleus is allowed to die in the egg, while in Rana it is completely removed. If a nucleus were to take up substances or fragments from the dying egg-nucleus of another subspecies, it might be expected to assimilate some of the characters of this other subspecies. A reversal of differentiation should be most easily discovered in nuclei derived from advanced 
donor stages, since these contain a greater proportion of nuclei which have lost their totipotency (Gurdon, Ig6oc). If such a reversal of differentiation does occur, it should be manifested by the expression of some " cytoplasmic" characters in transplant-embryos which were derived from hatched tadpole nuclei of one subspecies with the eggcytoplasm of the other. The tadpoles shown in text-fig. 2 (Ist and 5 th columns) are derived from tadpole nuclei of victorianus and eggcytoplasm of laevis, but in no case have they shown any laevis characters, either as embryos, larvae or adults. This negative result does not disprove the possibility of a reversal of differentiation, but merely constitutes some evidence against it. It confirms the belief that normal transplant-frogs derived from hatched tadpole gut cells represent entirely undifferentiated nuclei, so that the difference in rate of nuclear differentiation between Rana and Xenopus is real and not due to reversion or repair.

\section{(iii) The effect of serial transplantation on living nuclei}

The experiments described above provide a very critical test of whether nuclei are damaged by repeated transplantation and whether they continue to differentiate in spite of their renewed cytoplasmic environment. Since adult frogs capable of reproduction have been obtained after serial transplantation, a study of their development, growth and reproduction provides a wide opportunity for discovering in them any departure from normal. As described above, the frogs resulting from the fourth serial transfers were indistinguishable from controls in every respect including that of fertility and the development of their offspring. This shows that some nuclei at least are not in any way damaged by the technique, and that they do not undergo any additional differentiation during serial transfer. The absence of any tendency for nuclei to continue differentiation during serial transplantation provides a clear demonstration that the stimulus which initiates normal embryonic and nuclear differentiation must come in the first place from the cytoplasm. The evidence for the lack of technical damage applies not only to the somatic development brought about by transplanted nuclei, but also to the germ-line to which they give rise. This result will be of value as a control to further experiments with other more distantly related subspecies or species of Xenopus.

\section{SUMMARY}

Nuclei have been serially transplanted between two subspecies of Xenopus laevis. Donor nuclei were taken from embryos and tadpoles ranging in age from late blastulae to hatched tadpoles. The resulting transplant-embryos have been reared into adult frogs. These heterotypic embryos, tadpoles, and frogs show all the distinguishing characters of the subspecies which provided the nucleus, and none of those 
associated with the subspecies providing the egg-cytoplasm, except for characters directly dependent on the volume of recipient eggs.

Gastrula nuclei of $X$. l. victorianus were serially transferred four times into eggs of $X$. l. laevis, and gave rise to adult frogs which were indistinguishable from typical victorianus, and which were also capable of normal gametogenesis giving rise to offspring of victorianus type. Similarly, frogs derived from laevis nuclei transferred for one or two gastrula generations to victorianus cytoplasm, and those from nuclei transferred back to laevis cytoplasm after a gastrula generation in victorianus cytoplasm, were similar to control laevis frogs from fertilised eggs. The conclusions are that these nuclei were entirely unchanged after more than sixty divisions in foreign cytoplasm, and were likewise unaffected when returned to their own kind of cytoplasm after replication in that of the other subspecies.

The results also show that the serial transfer of nuclei has no tendency to diminish the viability or reproductive capacity of frogs derived from them. Lastly, the fact that some nuclei from advanced donor stages are unchanged after transfer to the eggs of the foreign subspecies indicates that these nuclei were undifferentiated at the time of transfer and have not undergone any reversal of developmental potentiality, such as might occur by assimilation of products of the irradiated egg-nucleus.

Acknowledgments.--The author is sincerely grateful to $\mathrm{Dr}$ M. Fischberg for his interest throughout this work, and to him and $\mathrm{Dr} \mathrm{A}$. W. Blackler for their most helpful criticism of the manuscript.

\section{REFERENCES}

astaurov, B. L., AND ostriakova-varshaver, v. P. I957. Complete heterospermic androgenesis in silkworms as a means for experimental analysis of the nucleus-cytoplasm problem. F. Embryol. exp. Morph., 5, 449-462.

BALTZER, F. I952. The behaviour of nuclei and cytoplasm in Amphibian interspecific crosses. Symp. Soc. exp. Biol., 6, 230-242.

BLACKLER, A. W., FISGHBERG, M., AND NEWTH, D. R., in preparation.

BRIGGS, R., AND KING, T. J. I953. Factors affecting the transplantability of nuclei of frog embryonic cells. F. exp. Zool., 122, 485-505.

BRIGGS, R., AND KING, T. J. I957. Changes in the nuclei of differentiating endoderm cells as revealed by nuclear transplantation. F. Morph., 10o, 269-312.

BRIGGS, R., AND KING, T. J. Ig6o. Nuclear transplantation studies on the early gastrula (Rana pipiens). I. Nuclei of presumptive endoderm. Devel. Biol., 2, 252-270.

DALTON, H. C. I946. The role of nucleus and cytoplasm in development of pigment patterns in Triturus. F. exp. Zool., 103, I69-199.

ELSDALE, T. R., FISGHBERG, M., AND SMITH, s, I958. A mutation that reduces nucleolar number in Xenopus laevis. Exp. Cell Res., 14, 642-643.

ELSDALE, T. R., GURDon, J. B., AND FISGHBERG, M. I96o. A description of the technique for nuclear transplantation in Xenopus laevis. F. Embryol. exp. Morph., $8,437-444$.

FISCHBERG, M., GURDON, J. B., AND ELSDAlE, T. R. 1958. Nuclear transfer in Amphibia and the problem of the potentialities of nuclei of differentiating tissues. Exp. Cell Res., Suppl. 6, I6 -1 78 . 
Plate IA

Photographs of selected stages in the embryonic and larval development of victorianus, laetis, and the reciprocal combinations of heterotypic transplant-embryos. Each photograph has been outlined in white, and melanophores where present have been darkened. 


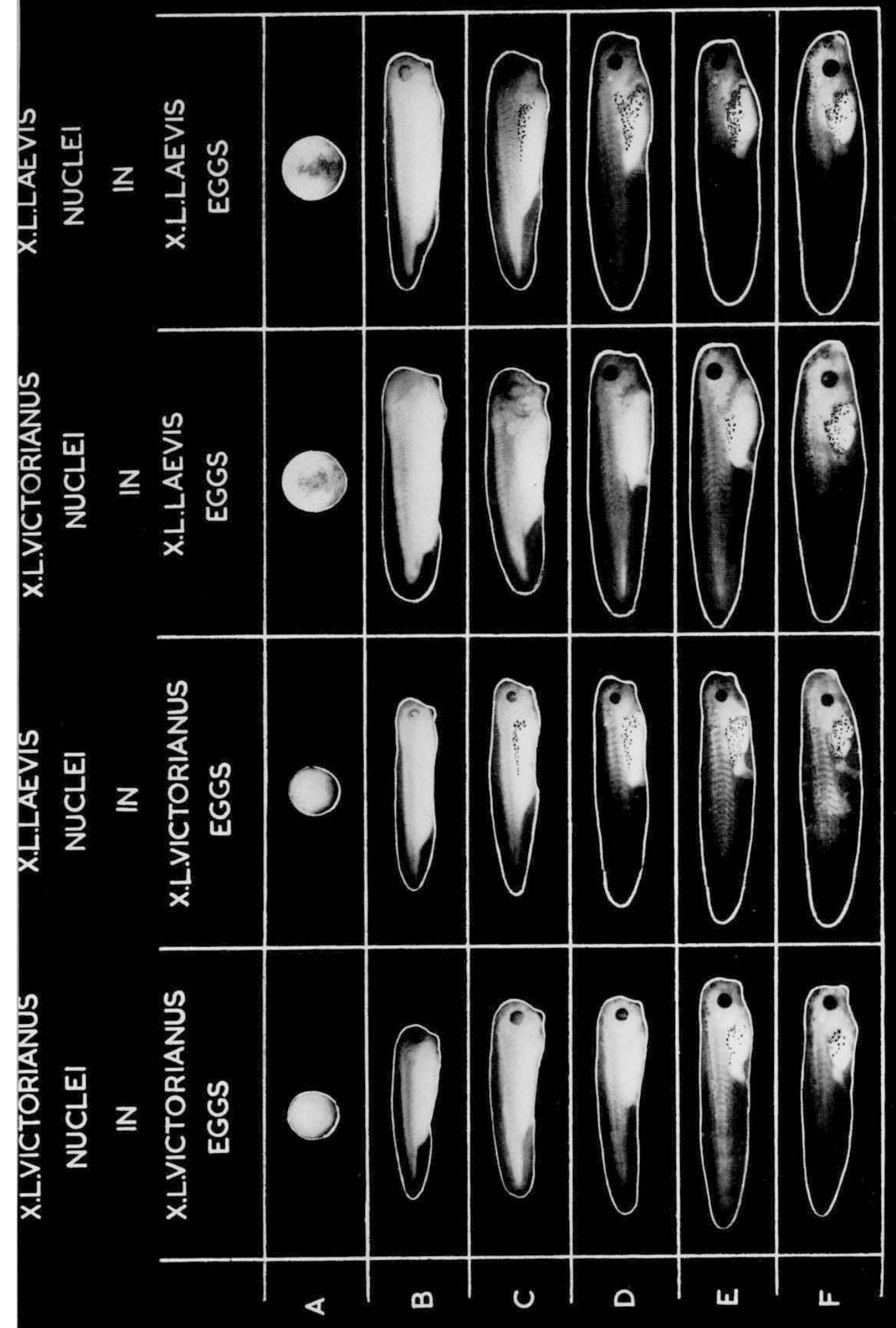


Plate IB

Photographs of adult frogs, all about one year old. The two frogs on the left are females. The remaining frogs are all males; the 3 transplant-frogs in the lower centre are derived from nuclei transferred to victorianus eggs and then back to laevis eggs. Gastrula donors were used for all serial transfers. 

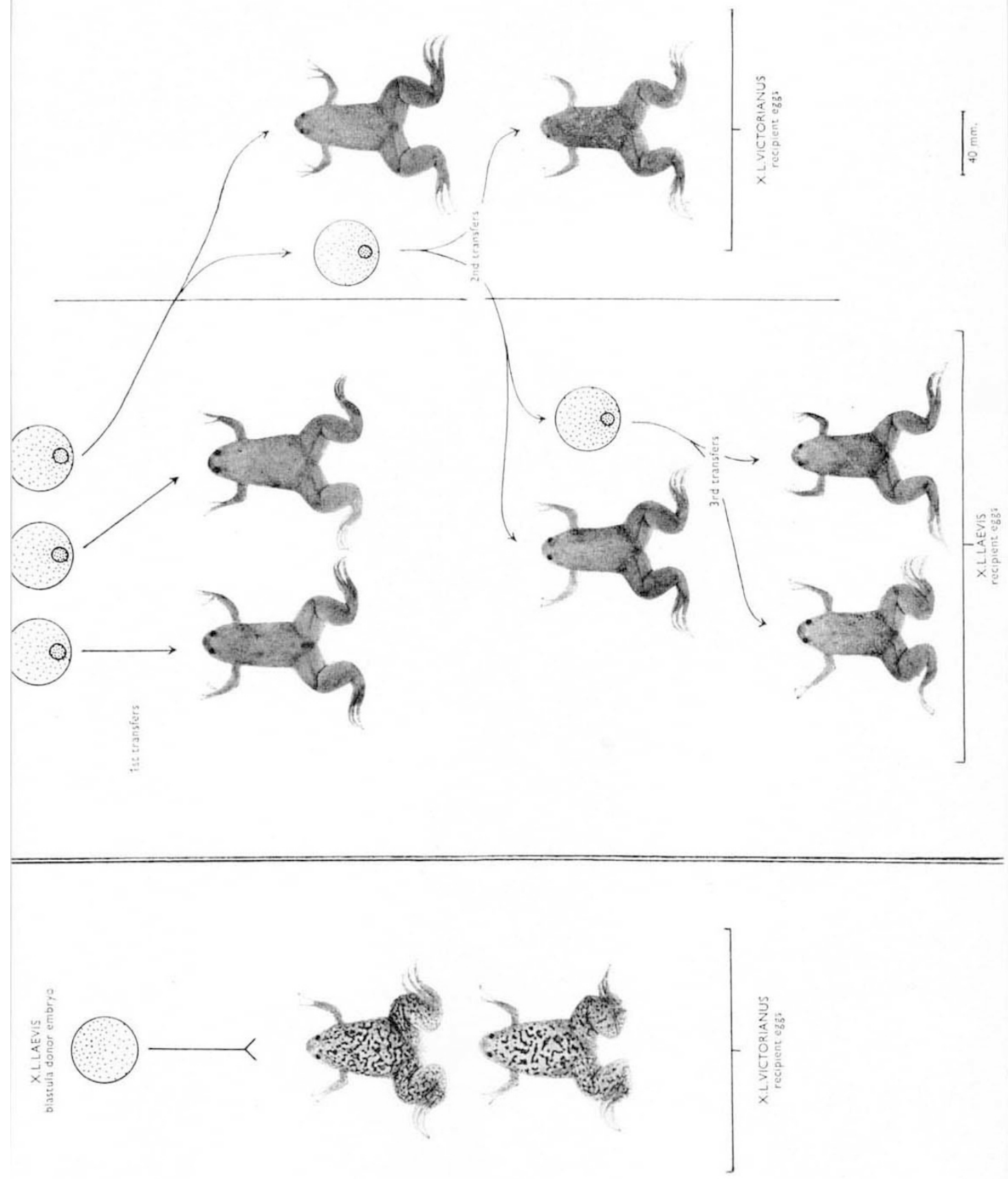


\section{Plate II}

Photographs of adult male frogs. All have been reared under laboratory conditions, and are between one and two years old. Also all frogs are derived from laeris eggcytoplasm. 
GURdon, J. B. I96oa. The effects of ultraviolet irradiation on the uncleaved eggs of Xenopus laevis. Quart. F. micr. Sci., IOI, 299-312.

GURDON, J. B. 196o $b$. Factors responsible for the abnormal development of embryos obtained by nuclear transplantation in Xenopus laeris. F. Embryol. exp. Morph., $8,327-340$.

GURDoN, J. B. 1960c. The developmental capacity of nuclei taken from differentiating endoderm cells of Xenopus laevis. 7. Embryol. exp. Morph., 8, 505-526.

GuRdon, J. B. 1961. Multiple genetically-identical frogs. 7. Hered., in press.

GURDON, J. B., ELSDALE, T. R., AND FISGHBERG, M. 1958. Sexually mature individuals of Xenopus laevis from the transplantation of single somatic nuclei. Nature, $182,64-65$.

HADORN, E. 1937. Die entwicklungsphysiologische Auswirkung der disharmonischen Kern-Plasmakombination beim bastardmerogon Triton palmatus (ㅇ) $\times$ Triton cristatus 0. Arch. Entw. Mech. Org., 136, 400-489.

Mckinnell, R. G. 196o. Transplantation of Rana pipiens (Kandiyohi dominant mutant) nuclei to $R$. pipiens cytoplasm. Amer. Nat., 94, 187-188.

MOORE, J. A. 1958. The transfer of haploid nuclei between Rana pipiens and Rana sylvatica. Exp. Cell Res. Suppl., 6, 179-191.

NIEUWKoop, P. D., AND FABER, J. 1956. A normal table of Xenopus laevis (Daudin). North-Holland Publishing Company, Amsterdam.

SAmBUichi, н. 1957. The roles of the nucleus and the cytoplasm in development I. An interspecific hybrid frog, developed from a combination of Rana nigromaculata nigromaculata cytoplasm with a diploid nucleus of Rana nigromaculata brevipoda. F. Sci. Hiroshima Univ. B.I., I7, 33-41. 